\title{
La cirugía brinda mayor alivio que el tratamiento conservador en pacientes con síndrome del túnel carpiano sin denervación
}

Surgery performs better than non-surgical therapy for carpal tunnel syndrome

\section{Objetivos}

Evaluar la mejoría funcional y sintomática en pacientes con túnel carpiano sin denervación, comparando cirugía y un tratamiento no quirúrgico multimodal (férula, ultrasonido e infiltración).

\section{Diseño, lugar y pacientes}

Ensayo clínico aleatorizado*, multicéntrico* (siete centros de EE.UU.) y simple ciego* (evaluador), con seguimiento final a un año. La aleatorización fue estratificada en bloque* por cada centro y la asignación se realizó en forma enmascarada* mediante sobre sellado.

\section{Intervención y medición de resultados}

Sobre un total de 116 pacientes, 57 fueron asignados a la rama de cirugía ( $49 \%$ mujeres, edad media 50 años) y 59 a la rama de tratamiento no quirúrgico (58\% mujeres, edad media 51 años).

El resultado principal se midió mediante la escala de estado funcional del Cuestionario de Evaluación del Síndrome del Túnel Carpiano ${ }^{1}$. Se definió como resultado exitoso a una disminución de 0,50 o más puntos, o $30 \%$ de mejoría del puntaje preoperatorio en el cuestionario. El análisis de los resultados se realizó por intención de tratar* y por protocolo*

\section{Resultados Principales}

Los resultados principales se detallan en la tabla 1. En cuanto a las variables intensidad del dolor, días laborales perdidos y cali-
Jarvik JG, y col. Lancet 2009;374:1074-81. dad de vida (formulario SF-36) no se observaron diferencias.

Tabla 1: Resultado primario a un año de seguimiento

\begin{tabular}{|c|c|c|c|}
\hline \multirow{2}{*}{ CESTC } & \multicolumn{2}{|c|}{12 meses de seguimiento ${ }^{8}$} & \multirow{2}{*}{$\begin{array}{l}\text { Efecto de tto* } \\
\text { (IC } 95 \%)\end{array}$} \\
\hline & $\begin{array}{c}\text { Tto quirúrgico } \\
n=49(\mathrm{DE})\end{array}$ & $\begin{array}{c}\text { Tto no quirúrgico } \\
n=52(\mathrm{DE})\end{array}$ & \\
\hline Estado funcional & $1,74(0,79)$ & $2,17(0,96)$ & $0,40(0,11$ a 0,70$)$ \\
\hline $\begin{array}{l}\text { Severidad de los } \\
\text { síntomas }\end{array}$ & $1,74(0,76)$ & $2,07(0,88)$ & $0,34(0,02$ a 0,65$)$ \\
\hline
\end{tabular}

CESTC: Cuestionario de Evaluación del Síndrome del Túnel Carpiano1. Tto: tratamiento.

*Indica la diferencia entre los grupos con tto quirúrgico y no quirúrgico, ajustado por el valor de base preoperatorio y el sitio de tratamiento.

\&: Por falta de datos al año, fueron excluidos ocho pacientes del grupo aleatorizado a cirugía y siete del grupo control.

\section{Conclusiones}

Ambos tratamientos mejoran los síntomas y la función a un año de seguimiento. Sin embargo, el tratamiento quirúrgico mejoró significativamente más los síntomas en los pacientes con síndrome del túnel carpiano sin denervación, que un tratamiento no quirúrgico bien definido.

Palabras claves: túnel carpiano, cirugía, ultrasonido.

Key words: carpal tunnel, surgery, ultrasound.

Fuente de financiamiento: National Institutes of Health $(\mathrm{NIH})$, National Institute of Artritis and Musculoskeletal and Skin Diseases, Intramural Research Program of the $\mathrm{NIH}$.

\section{Comentario}

En el presente trabajo ambos esquemas terapéuticos mostraron mejoría, sin embargo los pacientes asignados a cirugía tuvieron una mejoría significativamente mayor con respecto a los síntomas y a la función a los seis meses y al año de seguimiento. Esta diferencia fue mayor aún en el análisis por protocolo, lo cual se debe a una tasa de entrecruzamiento* del $39 \%$ hacia la rama quirúrgica. Existen algunos estudios con nivel de evidencia I y $\mathrm{II}^{*}$, que muestran que la cirugía brinda resultados superiores a otros tratamientos no quirúrgicos ${ }^{2-4}$. Aunque estos últimos tratamientos generan mejoría de los síntomas al igual que la cirugía, sus resultados no perduran en el tiempo ${ }^{3,5}$. Esto puede explicar la cifra de entrecruzamiento previamente señalada.

El reclutamiento de los pacientes se realizó en ocho establecimientos. Sin embargo, el $50 \%$ de los pacientes fueron reclutados en un solo centro. Esto último afecta y reduce el potencial de generalización de los resultados.

Aunque los autores sugieren que una fortaleza del estudio es la de utilizar un tratamiento no quirúrgico bien definido con intervención multimodal (ibuprofeno, ejercicios, ultrasonido), actualmente no hay evidencia sobre la eficacia de los AINES y los ejercicios en el tratamiento del túnel carpiano. Por otro lado, los estudios sobre la utilización del ultrasonido han mostrado efectividad ${ }^{6,7}$ en relación a la láser-terapia, aunque no se realizaron estudios contra un grupo de no tratamiento ${ }^{6}$. Debido a esto es que el ultrasonido tiene una recomendación grado $\mathrm{C}$ (opcional) por el grupo de estudio de la Asociación Americana de Cirujanos Ortopédicos ${ }^{8}$.

\section{Conclusiones del Comentador}

El tratamiento no quirúrgico en los pacientes con síndrome del túnel carpiano sin denervación es la opción inicial. Sin embargo, la falta de respuesta o la recidiva sintomática debe sugerir una liberación quirúrgica del nervio mediano para evitar los cambios irreversibles que produce la compresión nerviosa?.

Jorge Boretto [ Senvicio de Ortopedia y Traumatología del Hospital Italiano de Buenos Aires. jorge.boretto @ hospitalitaliano.org.ar ]

Boretto, J. La cirugía brinda mayor alivio que el tratamiento conservador en pacientes con síndrome del túnel carpiano sin denervación. Evid Act Pract Ambul 14(2):45. Abr-Jun 2011. Comentado de: Surgery versus non-surgical therapy for carpal tunnel syndrome: a randomized parallel-group trial. Jarvik JG, Comstock BA, Turner JA, et al. Lancet 2009; 374: 1074-81. PMID: 19782873.

\section{Referencias}

1.Levine D y col. A self-administered questionnaire for the assessment of severity of symptoms and functional status in carpal tunnel syndrome. J Bone Joint Surg Am 1993;75(11):1585-92. 2.Ucan $\mathrm{H}$ y col. Comparison of splinting, splinting plus local steroid injection and open carpal tunnel release outcomes in idiopathic carpal tunnel syndrome. Rheumatol Int 2006;27(1):45-51. 3.Demirci S y col. Comparison of open carpal tunnel release and local steroid treatment outcomes in idiopathic carpal tunnel syndrome. Rheumatol Int 2002;22(1):33-7. 4.Gerritsen A y col. Splinting vs surgery in the treatment of carpal tunnel syndrome: a randomized controlled trial. JAMA 2002;288(10):1245-51.

5.Hui A y col. A randomized controlled trial of surgery vs steroid injection for carpal tunnel syndrome. Neurology 2005;64(12):2074-8.

6.Bakhtiary A y col. Ultrasound and laser therapy in the treatment of carpal tunnel syndrome. Aust J Physiother2004;50(3):147-51.

7.O'Connor D y col. Non-surgical treatment (other than steroid injection) for carpal tunnel syndrome. Cochrane Database Syst Rev 2003;(1):CD003219.

8.Keith, M y col. American Academy of Orthopaedic Surgeons clinical practice guideline on the treatment of carpal tunnel syndrome. J Bone Joint Surg Am 2010;92(1):218-9. 9.Rempel, D y col. Pathophysiology of nerve compression syndromes: response of peripheral nerves to loading. J Bone Joint Surg Am 1999;81(11):1600-10. 